\title{
Eletroconvulsoterapia no tratamento da psicose puerperal
}

\author{
Electroconvulsive therapy in puerperal psychosis
}

Nuno Madeira ${ }^{1,2}$, Tiago Santos ${ }^{3}$, João Santos Relvas ${ }^{1,2}$, José Luís Pio Abreu ${ }^{1,2}$, Cristina Villares Oliveira ${ }^{1,2}$

\section{RESUMO}

A psicose puerperal (PP) é um quadro clínico exuberante, com uma frequência estimada de 1 caso por cada 1.000 puerpérios, e que tem sido associado com maior consistência ao espectro bipolar. A evidência científica disponível é escassa, nomeadamente no respeitante à orientação terapêutica. Os autores apresentam o caso clínico de uma mulher primípara de 28 anos, sem antecedentes psiquiátricos, que iniciou nas primeiras semanas de puerpério um quadro psicótico florido, composto por agitação psicomotora, confusão, desorganização do pensamento e comportamento, sintomatologia psicótica e insônia grave. Por ausência de resposta ao tratamento farmacológico, houve recurso à eletroconvulsoterapia (ECT), com melhoria clínica rápida e evidente. Nesse contexto, os autores realizaram uma apreciação da literatura e evidência referentes ao uso de ECT na psicose puerperal.

\section{ABSTRACT}

Puerperal psychosis (PP) is an exuberant clinical syndrome with an estimated frequency of 1 case per 1,000 childbirths that has been most consistently associated with the bipolar disorders spectrum. Available evidence is scarce, namely regarding management and treatment. The authors present the clinical case of a 28 year-old first-time mother, with no psychiatric history, who developed a florid psychotic syndrome in the first weeks of puerperium. Due to lack of improvement following pharmacological treatment, electroconvulsive therapy (ECT) was applied, with substantial and quick response. In this context, the authors reviewed current literature on the use of ECT for the treatment of puerperal psychosis.

\section{Keywords}

Electroconvulsive therapy, puerperal psychosis, postpartum psychosis.

Recebido em
$8 / 12 / 2011$
Aprovado em
$7 / 2 / 2012$

1 Hospitais da Universidade de Coimbra (HUC), Serviço de Psiquiatria.

2 Faculdade de Medicina da Universidade de Coimbra (FMUC).

3 Hospital Infante D. Pedro - Aveiro (HIP), Departamento de Psiquiatria e Saúde Mental.

Endereço para correspondência: Nuno Madeira

Serviço de Psiquiatria dos Hospitais,

Universidade de Coimbra Praceta Mota Pinto - 3000-075 - Coimbra, Portugal

Telefone: (351) 239-400-650/Telefax: (351) 239-403-950

E-mails: nunogmadeira@gmail.com; nunomadeira@huc.min-saude.pt 


\section{INTRODUÇÃO}

Por comparação com outras síndromes psiquiátricas do puerpério, a psicose puerperal (PP) é uma ocorrência relativamente infrequente, com incidência de 1 caso por 1.000 puerpérios ${ }^{1}$. O desenvolvimento de quadros psicóticos em subsequentes puerpérios é frequente, com recorrência estimada em $25 \%{ }^{2}$.

Uma arrastada controvérsia sobre a nosologia da psicose puerperal tem dado lugar à presunção de uma relação com a doença bipolar; admite-se também uma ligação com psicoses agudas polimórficas (classicamente descritas como "psicoses cicloides"), também elas potencialmente associadas ao espectro bipolar ${ }^{3}$. Um estudo epidemiológico conduzido por Kendell et al. documentou que $72 \%-80 \%$ das puérperas com PP teriam um diagnóstico de doença bipolar ou perturbação esquizoafetiva, e apenas $12 \%$ padeceriam de esquizofrenia'.

O precipitar de um episódio psicótico puerperal em muIheres com doença bipolar poderá, aliás, definir um subtipo de doença com relevância clínica e genética. Jones et al., investigando famílias com probandas bipolares e história de psicose puerperal, identificaram evidência sugestiva de linkage no cromossoma 16p134.

O início do quadro é, por regra, súbito, entre o $2^{\circ}$ e o $14^{\circ}$ dia de puerpério. São frequentes sintomas maniformes, psicóticos ou catatônicos; perplexidade e confusão são também comuns ${ }^{3}$. Além da desorganização do pensamento, comportamento bizarro, delírios autorreferenciais e persecutórios e ausência de insight, não é infrequente o registro de ideação suicida e homicida 5 .

De fato, a evolução do quadro clínico pode incorrer em consequências potencialmente nefastas: hospitalização da mãe - com potencial prejuízo da vinculação e desenvolvimento do lactente, risco de autoagressão ou suicídio ${ }^{6}$, e raros, mas dramáticos, casos de dano ao recém-nascido, nomeadamente infanticídio?. A psicose puerperal grave deverá, pois, ser considerada uma urgência psiquiátrica, sendo necessários intervenção e tratamento imediatos.

Embora não existam guidelines terapêuticas para a abordagem da PP, o tratamento de primeira linha habitual consiste na utilização de antipsicóticos e estabilizadores de humor ${ }^{8}$. No entanto, o pleno efeito da farmacoterapia não é imediato, e a quase totalidade dos psicofármacos é excretada no leite materno, pelo que é recomendada, à maioria das mulheres, a suspensão da amamentação, em favor do tratamento farmacológico?.

\section{RELATO DE CASO}

L., caucasiana de 28 anos, foi internada no Serviço de Psiquiatria dos Hospitais da Universidade de Coimbra duas semanas após o nascimento do seu primeiro filho, na sequência de vinda ao Serviço de Urgência por quadro de irritabilidade, agitação psicomotora e insônia grave. O parto, por cesariana, decorrera sem complicações, bem como a gestação, vigiada e sem intercorrências. Não se documentou história pessoal ou familiar de doença psiquiátrica.

No internamento, o quadro clínico agravou-se rapidamente, apresentando-se a doente confusa e perplexa, com discurso incoerente e ideias delirantes paranoides e de carácter místico. A investigação laboratorial (hemograma, bioquímica básica, urina sumária, provas tireoideas e serologias) revelou-se negativa; os demais exames realizados - tomografia axial computorizada cranioencefálica e eletroencefalograma - revelaram-se normais. A doente foi inicialmente medicada com risperidona (4 mg/dia) e depois haloperidol (10 mg/dia), substituídos após desenvolvimento de sintomatologia extrapiramidal. Não se verificou melhoria clínica com outros antipsicóticos (ziprasidona 160 mg/dia, olanzapina 30 mg/dia), em associação com benzodiazepinas (lorazepam $10 \mathrm{mg} / \mathrm{dia}$ ). Foi ainda tentada introdução de carbamazepina (400 mg/dia), que veio a ser suspensa por rash cutâneo. Por causa da gravidade do quadro clínico e da profusa medicação psicotrópica empregada, foi suspensa na primeira semana de internamento a amamentação.

Após um mês de internamento, optou-se pelo recurso à eletroconvulsoterapia por falha da abordagem psicofarmacológica. Nas sessões de ECT, foi utilizado um aparelho MECTA SpECTrum 5000Q, com monitorização de parâmetros vitais (Diascope Traveller da S\&W). Foi empregada estimulação bilateral, com eletrodos temporais. Como anestésico, utilizou-se propofol (doses por sessão compreendidas entre 110 e $150 \mathrm{mg}$ ), sendo a succinilcolina (entre 25 e $40 \mathrm{mg}$ ) o relaxante muscular usado. As baixas doses de curarizante se relacionam com o método de monitorização das crises convulsivas: na data nosso equipamento de ECT não dispunha de monitor eletroencefalográfico, pelo que a utilização de doses mais reduzidas de succinilcolina permitiu a monitorização pelo método eletromiográfico modificado. Como critério de adequação terapêutica, foi utilizada a duração da crise (necessariamente superior a 20 s). Para doseamento do estímulo elétrico, recorreu-se ao método de dose fixa (pré-selecionada) no primeiro tratamento, com ajustamentos sucessivos nos tratamentos seguintes em função da duração da crise e da resposta clínica.

Houve resposta clara e marcada após as primeiras duas sessões, encontrando-se a doente em quase remissão após uma semana de tratamento. Foram realizadas sete sessões de ECT, regressando a doente ao domicílio após três semanas, medicada com olanzapina 10 mg/dia e levomepromazina $50 \mathrm{mg} / \mathrm{dia}$. Realizou-se uma sessão adicional de ECT em ambulatório. A evolução posterior foi favorável: após três meses foi suspensa a medicação psicotrópica, permanecendo a doente assintomática há três anos. O seu filho 
tem tido uma infância saudável, com ótimo desenvolvimento psicomotor.

\section{DISCUSSÃO}

A eletroconvulsoterapia, empregada com sucesso no caso clínico relatado, foi sugerida como sendo extremamente eficaz no tratamento da psicose puerperal, em particular nos casos refratários a tratamentos somáticos convencionais ${ }^{10}$.

A rapidez e a eficácia da intervenção por ECT podem ser determinantes para que se desenvolva uma adequada vinculação mãe-lactente, reduzindo a duração da descompensação psicótica e do internamento, frequentemente necessária em quadros de PP. De fato, uma disrupção precoce da relação mãe-lactente associa-se a consequências adversas para a criança a curto e longo prazo ${ }^{11}$.

Outro benefício do recurso à ECT prende-se com a possibilidade de permitir à mãe prosseguir com a amamentação. Uma significativa proporção dos psicofármacos é segregada no leite materno, nem sempre existindo dados quanto ao seu efeito imediato e a longo prazo no desenvolvimento dos lactentes. Os benefícios da amamentação estão amplamente reconhecidos; segundo a Organização Mundial de Saúde (OMS) e a agência United Nations Children's Fund (Unicef), o máximo benefício da saúde e desenvolvimento dos lactentes consagra a amamentação exclusiva nos primeiros quatro a seis meses de vida'2.

$\mathrm{Na}$ eletroconvulsoterapia, o uso de agentes anestésicos seria uma limitação potencial à amamentação. Um estudo envolvendo fármacos comumente utilizados na indução anestésica (propofol, midazolam e fentanil) documentou doses máximas no lactente inferiores a 0,1\% da dose administrada - bem abaixo do limiar clínico de significância da exposição de lactentes a fármacos segregados no leite materno ${ }^{13}$. As recomendações atuais não preconizam, pois, a interrupção da amamentação após a recuperação pós-anestésica?.

No entanto, apesar da impressão clínica quanto à ECT como tratamento de escolha na psicose puerperal grave e refratária, existe escassa evidência na literatura referente a essa prática, assinalando-se a ausência de ensaios clínicos randomizados e controlados. A esse respeito, com objetivo de revisar a evidência disponível relativa ao uso de ECT no tratamento da psicose puerperal, foi efetuada uma pesquisa, sem limite de data, nas bases PubMed/Medline, utilizando os termos de pesquisa "puerperal/postpartum psychosis" e "electroconvulsive therapy/ECT". Dessa pesquisa, obtiveram-se somente duas publicações, entre as quais o trabalho de Reed et al., um estudo retrospectivo visando comparar a eficácia da ECT em psicoses puerperais, em mulheres com outros quadros psicóticos; os autores concluíram por uma particular eficácia desse tratamento somático na mulher puérpera ${ }^{14}$. Mais recentemente, Forray e Ostroff publicaram uma série de cinco casos de de- pressão ou psicose puerperal, refratárias à intervenção farmacológica, em que a ECT foi utilizada com manifesto sucesso?.

O principal efeito secundário descrito, tradicionalmente associado à ECT, é a amnésia transitória. Têm sido sugeridas estratégias para minimizar as queixas mnésicas, como o uso de eletrodo unilateral, ou o recurso à ketamina como agente anestésico ${ }^{15}$.

\section{CONCLUSÃO}

A psicose puerperal, pelos riscos implícitos para a mãe e lactente (prejuízo da vinculação, suicídio, infanticídio), exige um manejo rápido e eficiente.

A eletroconvulsoterapia, utilizada com sucesso no caso clínico reportado, tem sido descrita na literatura como uma opção relevante no tratamento dos quadros psicóticos do puerpério, com efeito rápido e robusto. Permite minimizar o período sintomático e restabelecer tão cedo quanto possível a normalidade da relação mãe-lactente, além de possibilitar a prossecução da amamentação materna.

Embora não existam ensaios clínicos randomizados e controlados que documentem a validade da intervenção, o caso clínico reportado e a evidência disponível em outros relatos de casos e estudos retrospectivos defendem o uso da eletroconvulsoterapia como escolha no tratamento da psicose puerperal, quer refratária à terapêutica farmacológica quer de gravidade significativa, por exemplo, em caso de ameaça à integridade da mãe e lactente.

Será necessária investigação futura que consolide o benefício dessa intervenção terapêutica, nomeadamente ensaios controlados e randomizados que avaliem a eficácia da ECT na psicose puerperal quando comparada com a terapêutica farmacológica.

\section{CONFLITOS DE INTERESSE}

Os autores declaram não ter nenhum conflito de interesses relativo ao presente artigo. Não existiram fontes externas de financiamento para a realização deste artigo.

\section{REFERÊNCIAS}

1. Kendell RE, Chalmers JC, Platz C. Epidemiology of puerperal psychoses. Br J Psychiary. 1987;150:662-73.

2. Brockington I. Postpartum psychiatric disorders. Lancet. 2004;363:303-10.

3. Brockington I. Obstetric and gynaecological conditions associated with psychiatric disorder. In: Gelder M, Andreasen N, López-Ibor J, Geddes J (editors). New Oxford Textbook of Psychiatry. New York: Oxford University Press; 2009. p. 1114-27.

4. Jones I, Hamshere M, Nangle JM, Bennett P, Green E, Heron J, et al. Bipolar affective puerperal psychosis: genome-wide significant evidence for linkage to chromosome 16. Am J Psychiatry. 2007;164:1099-104. 
5. Spinelli MG. Postpartum psychosis: detection of risk and management. Am J Psychiatry. 2009;166(4):405-8.

6. Appleby L, Mortensen PB, Faragher EB. Suicide and other causes of mortality after post-partum psychiatric admission. Br J Psychiatry. 1998;173:209-11.

7. Spinelli MG. A systematic investigation of 16 cases of neonaticide. Am J Psychiatry. 2001;158(5):811-3.

8. Dennis $\mathrm{CL}$, Stewart DE. Treatment of postpartum depression, part 1: a critical review of biological interventions. J Clin Psychiatry. 2004;65(9):1242-51.

9. Forray $A, 0$ stroff RB. The Use of electroconvulsive therapy in postpartum affective disorders. J ECT. 2007;23(3):188-93.

10. Rabheru K. The use of electroconvulsive therapy in special patient populations. Can J Psychiatry. 2001;46(8):710-9.
11. Murray L, Sinclair D, Cooper P, Ducournau P, Turner P, Stein A. The socioeconomical development of 5-year-old children of postnatally depressed mothers. J Child Psychol Psychiat. 1999;40(8):1259-71.

12. World Health Organization/United Nations Children's Fund. The Innocenti declaration on the protection, promotion and support of breastfeeding. Disponivel em: http://www.unicef.org/programme/breastfeeding/innocenti.htm. Acesso em: Nov 1, 2011.

13. Nitsun M, Szokol JW, Saleh HJ, Murphy GS, Vender JS, Luong L, et al. Pharmacokinetics of midazolam, propofol, and fentanyl transfer to human breast milk. Clin Pharmacol Ther. 2006;79(6):549-57.

14. Reed P, Sermin N, Appleby L, Faragher B. A comparison of clinical response to electroconvulsive therapy in puerperal and non-puerperal psychoses. J Affect Disord. 1999;54(3):255-60.

15. McDaniel W, Sahota A, Vyas BV, Laquerta N, Hategan L, Oswald J. Ketamine appears associated with better word recall than etomidate after a course of 6 electroconvulsive therapies. J ECT. 2006;22(2):103-6. 
Na edição de 2012;61(1):45-8, no artigo "Eletroconvulsoterapia no tratamento da psicose puerperal", onde se lê:

Nuno Madeira', Tiago Santos², José Pio Abreu33, Cristina Villares Oliveira

1 Universidade de Coimbra (HUC), Serviço de Psiquiatria dos Hospitais.

2 Hospital Infante D. Pedro - Aveiro (HIP), Departamento de Psiquiatria e Saúde Mental.

3 Faculdade de Medicina da HUC, Serviço de Psiquiatria.

\section{Leia-se:}

Nuno Madeira ${ }^{1,2}$, Tiago Santos ${ }^{3}$, João Santos Relvas ${ }^{1,2}$, José Luís Pio Abreu ${ }^{1,2}$, Cristina Villares Oliveira ${ }^{1,2}$

1 Hospitais da Universidade de Coimbra (HUC), Serviço de Psiquiatria.

2 Faculdade de Medicina da Universidade de Coimbra (FMUC).

3 Hospital Infante D. Pedro - Aveiro (HIP), Departamento de Psiquiatria e Saúde Mental. 